\title{
Epidemiological treatment of venereal disease contacts
}

\author{
WILLIAM J. BROWN \\ Georgia Department of Human Resources, Division of Physical Health, Atlanta, Georgia, U.S.A.
}

The resurgence of both gonorrhoea and infectious syphilis in recent years serves as a reminder that control efforts directed against these diseases must be maintained and strengthened rather than relaxed. It is certain that the epidemiological treatment of the contacts of cases of infectious syphilis is the most powerful preventive weapon in the hands of both public health and private physicians. Furthermore, there is ample evidence that epidemiological treatment of the contacts of men with gonorrhoea is also essential in checking the spread of this disease.

\section{Syphilis}

Some 30 per cent. of the contacts of cases of infectious syphilis will themselves develop syphilis, but unfortunately there is no method of predicting which exposed individuals will develop the disease. Withholding treatment and keeping the contact under observation while waiting for the lesions of syphilis to appear or seroconversion to occur, although perhaps theoretically appealing, is beset by many difficulties in practical application. In the first place, all too often patients do not return for followup examinations. Secondly, because the infectious lesions of syphilis may be present before seroconversion, follow-up examinations would have to include a careful inspection of all mucous membrane surfaces, including the rectum in females and homosexual males, thus requiring proctoscopy; moreover, any questionable lesion on any part of the body developing during the 90 days after exposure would have to receive a darkfield examination. Finally, even if the contact returns monthly for such physical and serological examinations, the infectious lesions may develop on the day after the examination, so that, assuming that the suspects do not abstain from intercourse during the period of followup, another potential chain of transmission may be started.

The files of the Venereal Disease Branch, Centre for Disease Control, are replete with examples of epidemics which started as a result of the substitution of 'watchful waiting' and observation of contacts for the prompt epidemiological treatment of such contacts. An illustration of such a documented epidemic is presented below.

A private physician reported a case of primary syphilis in a 28-year-old man. When interviewed by a trained public health worker, the man named seven widespread contacts with whom he had had intercourse since the primary lesion had appeared. Of these, two were residents of a neighbouring state where they were examined and given preventive treatment. The remaining five (one of whom was the patient's own wife) were local residents. All five were examined and none showed either lesions of syphilis or a reactive serological test for syphilis. Unfortunately, the local health officer did not believe in epidemiological treatment as a preventive measure. Upon follow-up 30 days later, three of the five, including the patient's wife, had developed infectious syphilis and the fire igniting the epidemic had been set. Before it was brought under control, 25 persons had been infected with syphilis, all of whom should have been spared this unfortunate happening. Although a little late, the health officer became a 'believer' when shown what had developed because of his unwillingness to utilize the best case prevention weapon at his disposal.

Another great advantage of a programme of vigorously applied epidemiological treatment is that all sexual partners in a group are treated within a short period of time, thus avoiding the re-infection of a previously treated patient in 'ping-pong' fashion. An example of this phenomenon is given below.

A 24-year-old man sought medical attention in a hospital emergency room because of a penile lesion of several weeks' duration. He was given therapy at that time, but his wife had a non-reactive serological examination and no history of lesions and did not receive epidemiological treatment. The husband returned to the emergency room with another primary lesion 4 months later, his only sexual contact during the entire period having been his wife. Interviews revealed she too had had no other sexual contacts, but examination showed reactive serologicai tests and the characteristic lesions of secondary syphilis. She had first been infected by her husband when he had primary syphilis 4 months earlier, and 
at the time of her previous examination she had had an incubating syphilitic infection which could not be detected. Her syphilitic lesions during the secondary stage of the disease had then been responsible for the 'ping-pong' infection back to her husband.

With the institution of a vigorous epidemiological treatment programme the State of Alabama has seen the incidence of primary and secondary syphilis drop from 119 cases per month in 1965 to seven per month recently, with most of these cases traceable to 'imported' syphilis from other states. During this same period the infectious syphilis case rate in Alabama dropped from being the highest in the United States to the 30th. Nationally, it is estimated that 2,500 cases of primary and secondary syphilis are prevented yearly through the use of epidemiological treatment.

\section{Gonorrhoea}

Similarly, a strong case can be made out for the epidemiological treatment of the contacts of male patients with gonorrhoea. Culture surveys performed by the Venereal Disease Branch indicate that 37 per cent. of women named as contacts by infected male patients will have positive cervical cultures at the first examination. Because of the difficulties en- countered in following up such patients, it is irresponsible not to administer therapy to these women. Although extensive data are not available on the infection rate among male contacts of men known to have urethral gonorrhoea, indirect evidence suggests these too are at high risk and should receive epidemiological treatment.

\section{Concomitant syphilis}

There is the added advantage that the doses of penicillin currently employed for the therapy of gonorrhoea $(4.8$ m.u. aqueous procaine penicillin $\mathrm{G}$ with $1 \mathrm{~g}$. probenecid) are known to be more than adequate for aborting concomitant incubating syphilis. This makes a lengthy and expensive follow-up for syphilis unnecessary in this group.

\section{Conclusions}

With the increasing mobility of the 15 to 25 -yearolds who comprise the age group at greatest risk of acquiring venereal disease, adequate follow-up examinations are becoming more difficult to achieve. Thus the epidemiological treatment of contacts, already the keystone in venereal disease control, will become even more important in the future. 